\title{
Sequential nitrification and denitrification in a novel palm shell granular activated carbon twin-chamber upflow bio-electrochemical reactor for treating ammonium-rich wastewater
}

Introduction

The uncontrolled discharge of nitrogen components through nitrogen-rich wastewaters has been considered as a worldwide human health threat and environment challenge (Chen et al., 2006).

In wastewater treatment plants with an anaerobic digester, reject water from dewatering digested sludge contains a significant amount of nitrogen (15-25\% of the total nitrogen load in influent), which the effluent recycles to the head of the sewage treatment works (Mata-Alvarez, 2002; Dosta et al., 2007). The specific characteristics of the reject water, such as high concentration of ammonium and low $\mathrm{C} / \mathrm{N}$ ratio, allow the application of special treatment methods. A separate treatment of reject water would significantly increase the elimination of nitrogen by decreasing the nitrogen load in the main stream (Fux et al., 2002).

Biological methods are more cost effective compared with physical-chemical techniques. In a conventional approach, nitrogen elimination is done through sequential nitrification and heterotrophic denitrification. The low ratio of $\mathrm{C} / \mathrm{N}$ in reject water and high operational costs, attributed to $\mathrm{O}_{2}$ provision and organic carbon necessities, have impeded broad implementation of conventional nitrification and denitrification. Recently, new methods, such as biological nitrogen removal via nitrite, have been applied as effective and economical approaches in treating reject water (Jenicek et al., 2007). Biological nitrogen removal is performed via nitrite due to the lower oxygen requirement (25\%) and chemical oxygen demand (COD) $40 \%$ for denitrification, as well as faster kinetic of the process, in contrast with removal via nitrate. In addition, heterotrophic denitrification is deemed unfavorable (Zhao et al., 2011) due to limitations such as residual carbon sources, by-products that incur further post-treatment costs, clogging in reactors, and increases the effluent turbidity because of high growth rate. Therefore, researchers have used autotrophic bacteria; using inorganic compounds as carbon sources $\left(\mathrm{CO}_{2}\right.$ and $\mathrm{NaHCO}_{3}$ ), is less expensive compared with using organic sources and has harmless residuals. On the other hand, autotrophic bacteria use inorganic compounds, such as manganese, hydrogen sulfide, ferrous iron, and molecular hydrogen as energy sources 
(Ghafari et al., 2008; Mousavi et al., 2012). Among these compounds,

$\mathrm{H}_{2}$ is a perfect alternative to alleviate the previously mentioned

problems due to its cleanliness. In addition, $\mathrm{H}_{2}$ is the best

electron donor among other elements (Mousavi et al., 2012). Consequently,

bacteria that use hydrogen as energy source, namely,

"autohydrogenotrophic bacteria," have been considered for the

denitrification process. The application of autohydrogenotrophs

in reactors requires proper methods of supplying hydrogen to the

microorganisms. The initial injection of hydrogen gas into the reactor

is a method with certain limits (Lee and Rittmann, 2002), such

as low solubility of $\mathrm{H}_{2}$ in water ( $1.6 \mathrm{mg} \mathrm{L} \mathrm{L}_{-} 1$ at $20 \_\mathrm{C}$ ). This limitation

declines the accessibility and raises the contact time for the

biological process. Consequently, this also causes a decrease in

the denitrification rate compared with heterotrophic denitrification

(Prosnansky et al., 2002; Vasiliadou et al., 2006). Some marginal

concerns are also reported in the application of hydrogen,

such as the costly supply of $\mathrm{H}_{2}$, flammability, as well as transport

and storage problems (Ghafari et al., 2008). Thus, in situ hydrogen

generation (water electrolysis) is recommended as an efficient

technique to overcome these drawbacks. Therefore, researchers

have considered a new integrated system namely bio-electrochemical

reactor $(B E R)$ to treat water and wastewater.

A BER is defined as a system that consists of integrated processes

(biological and chemical processes), which by electrolysis

of aqueous solution prepare desirable oxic and anoxic conditions

for microorganisms by passes the electric current through electrodes

due to the production of suitable electron donors $\left(\mathrm{H}_{2}\right)$ and

electron acceptors $\left(\mathrm{O}_{2}\right)$ as expressed in Eqs. (1) and (2) (Ramesh

and Goel, 2005).

Please refer to the full text

A comprehensive review, recently by authors showed mainly

previous studies have focused on eliminating nitrate via BERs

(Mousavi et al., 2012) whereas, some researchers have applied this

system for simultaneous or sequential nitrification and denitrification

(SND) in different configurations. Watanabe et al. (2002) studied

the possibility of simultaneous nitrification and denitrification

in a single bio-electrochemical reactor. The single reactor enhances

aerobic degradation by the production of oxygen in the anode

zone, but adversely influences the anaerobic microorganism

(Watanabe et al., 2002). Therefore, researchers have tried to establish

a new configuration of BER as divided cell, which prepare the

overall capacity of a BER without harmful effect of generated $\mathrm{O}_{2}$ in

anode on anoxic condition in cathode zone (Szekeres et al., 2001;

Ramesh and Goel, 2005). In addition, researchers have attempted

to speed up the biological treatment by finding ways to provide 
better contact between the substrate and microorganisms via the immobilization of the biomass at the surface of the electrodes.

The granular activated carbon (GAC), due to many advantages such as low cost, durable packing material, enhancement of the adhesion of bacteria and large macropore volume (Moreno-Castilla et al., 2003; Jiang and Li, 2009), has been applied in different studies as biocarrier and electrode to provide the surface for the creation of biofilm and increase the oxygen and hydrogen gas yield. Moreover, the third bipolar electrode produces carbon dioxide to provide a desirable anoxic condition (Zhang et al., 2005; Zhou et al., 2007; Ghafari et al., 2009).

Fully autotrophic nitrification and denitrification in a palm shell granular activated carbon twin-chamber upflow bio-electrochemical reactor (PSGAC-TCUBER) has neither been documented nor implemented in previous studies. Therefore, the aims of the present study were to investigate the effect of hydraulic retention time (HRT) and electric current (I) on nitrogen removal and to optimize the operating parameters of the reactor for SND. In this regard, the central composite design (CCD) is a Design-Expert software used in all of the experiments. The developed polynomial models analyzed the data obtained from different runs. The sufficiency of the models was investigated via analysis of variance (ANOVA) to provide the statistical findings and diagnostic checking tests of the models.

\section{Methods}

\subsection{Experimental set-up}

The PSGAC-TCUBERs were built from Plexiglas tubes as shown in the schematic diagram of the experimental apparatus in Fig. 1. The reactors provided the desired oxic and anoxic conditions at the anode and cathode zones, respectively, using electric intensity in the divided cell. The reactor was designed to consist of two columns joined together by a connection (Plexiglas tubes: $8 \mathrm{~cm}$ long and $6 \mathrm{~cm}$ in internal diameter) located $4 \mathrm{~cm}$ from the bottom. The adjoining parts separated the anode and cathode compartments from each other by a cation permeable membrane (CPM) (Nafion 117, Dupont Wilmington, DE) through a flange and O-ring type joint. Each column was $42 \mathrm{~cm}$ in height and had an inner diameter of $10 \mathrm{~cm}$. A mesh plate stainless steel (type 316) as electrodes (hole size $1_{-} 1 \mathrm{~mm}$ and $0.1 \mathrm{~cm}$ thickness) was installed at the bottom of each column and screwed to the plates, 12 stainless steel rods (5 $\mathrm{mm}$ in diameter and $40 \mathrm{~mm}$ in height) to enhance the supply of electricity in the palm shell-GAC as anode and cathode zones. The copper wires, which were sealed with fast-drying epoxy coated with silicon sealant, were connected to the electrodes 
through the current collectors. The wires were connected directly to a programmable DC power supply (IPS-3202, RS Components, England). The reactors were watertight at the bottom and top using O-ring flanges to prevent the transfer of atmospheric gases in anaerobic and anoxic zones. The $600 \mathrm{~g}$ PSGAC from Bravo Green Sdn. Bhd., Sarawak, Malaysia with a size of 2-4 mm was packed into both columns (anode and cathode) with a height of $22 \mathrm{~cm}$ as biocarrier and third electrode. The total volume of the reactor in each compartment was $3.5 \mathrm{~L}$. The effluent ports were located $33 \mathrm{~cm}$ above the plate in the nitrification and denitrification chambers. The working volume for each column was $1.8 \mathrm{~L}$, and the effective volume of $1 \mathrm{~L}$ was considered as anode and cathode zones. The void volume at the electrode zone was $1 \mathrm{~L}$, and the ratio was 0.57 . A number of sampling ports (SP) were installed along the opposite side of each column to inoculate the sludge on the PSGAC. Sampling ports played an important role in adjusting the $\mathrm{pH}$ between

7.6 and 8.0 via an automatic injection of acid $\left(\mathrm{H}_{2} \mathrm{SO}_{4} ; 1 \mathrm{~N}\right)$ or alkaline $(\mathrm{NaOH} ; 1 \mathrm{~N})$ solution through a pH controller (Biostat_B, B.

Braun, Biotech International), which was connected to fermenter. A reverse cone-shaped funnel was used as a gas separator to transfer the produced gases through the outlet to the water seal. The controlling parameters ( $\mathrm{DO}$ and $\mathrm{pH}$ ) were monitored using $\mathrm{DO}$ meter (METTLER TOLEDO, O2-sensor, Switzerland) and pH meter (METTLER TOLEDO, ph-sensor, Switzerland) positioned at a height of $25 \mathrm{~cm}$ from the bottom of the reactors in both compartments.

2.2. Inoculated biomass and feeding

The mixed culture of acclimatized nitrifying bacteria and hydrogenothrophic denitrifying bacteria from prior research studies 


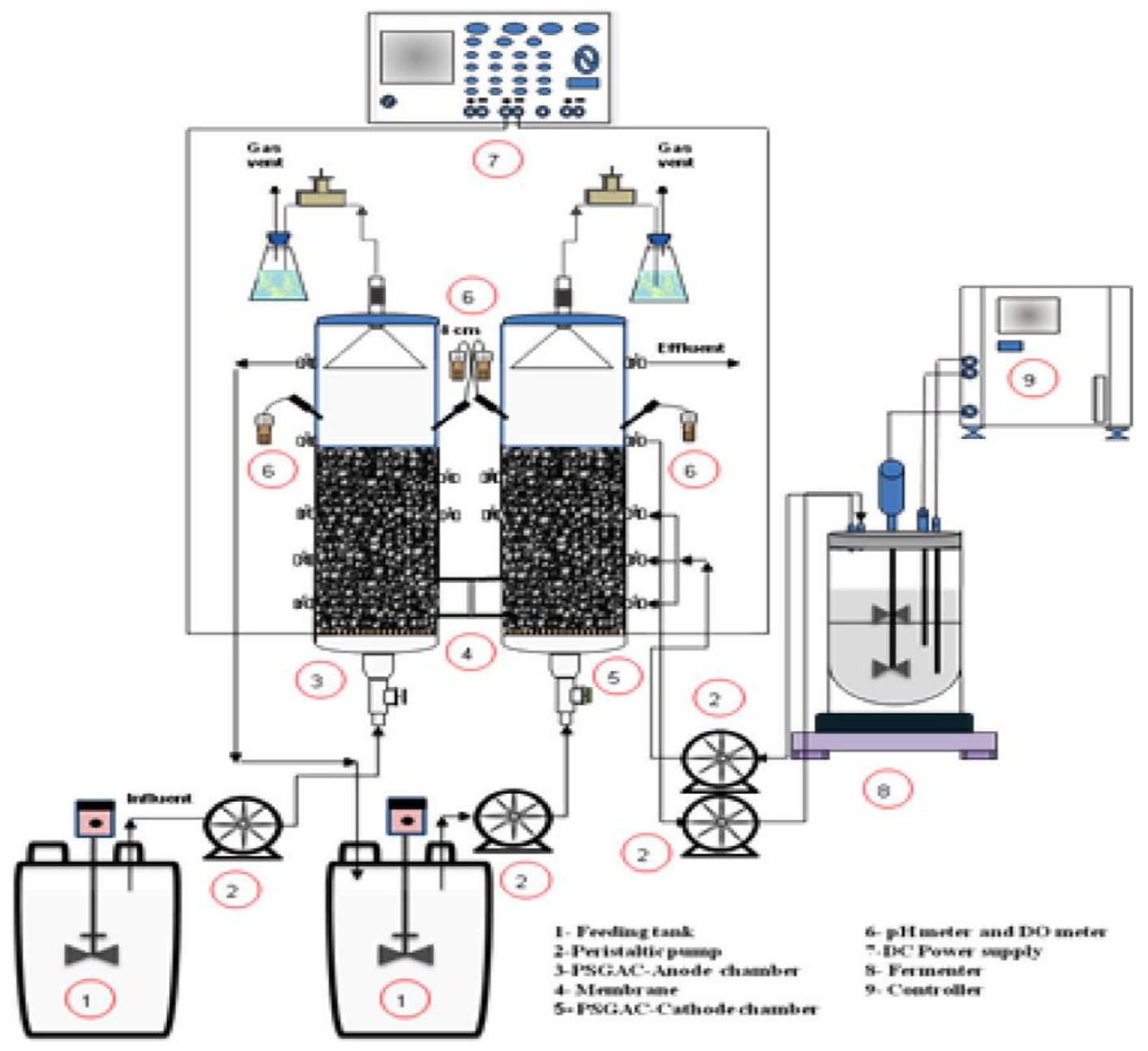

Fig. 1. Schematic of palm shell granular activated carbon twin chamber up-flow bio-electrochemical reactor (PSGAC-TCUBER).

inoculated into the TCUBERs after 20 days of re-acclimatization, to

grow on the PSGAC. Before the injection of biomass inside the reactors,

the following preparation of bio-carrier was performed.

The washed PSGAC was soaked several times in deionized water

overnight. The deionized water was drained from the GAC, and then

dried at 105 _C. The GAC was then saturated in 1\% agar solution

(Bacto ${ }^{\mathrm{TM}}$ Agar) at boiling temperature to produce a sticky surface.

The cold PSGAC was transferred into BERs, and the reacclimatized

sludge, with initial mixed liquor suspended solids (MLSS)

(3,000 mg L_ 1 ), was pumped on the GAC columns through five sampling

ports from the opposite sides of each compartment. The volumetric

ratio of GAC to total reactor volume was 0.55 . The growth and

re-acclimatization of biomass on the GAC was maintained for

40 days by controlling the environmental and operational parameters:

$\mathrm{pH}$ of $7.8 \pm 0.2 ; \mathrm{T}$ of $29 \pm 1 \_\mathrm{C} ; \mathrm{NH}_{4}$

$+-\mathrm{N}$ of $200 \mathrm{mg} \mathrm{L}$ 
The BERs from the oxic part (anode compartment) was fed with

synthetic wastewater according to Table 1. The anoxic part (cathode

compartments) was fed with effluent from the first compartment

(anode). To limit the interference and unsuitable effect of

$\mathrm{O}_{2}$ on the denitrification process, the nitrogen gas was sparged

through an influent at a rate of $2 \mathrm{~L}$ min_1 for $5 \mathrm{~min}$ to drive off

the $\mathrm{DO}\left(<0.5 \mathrm{mg} \mathrm{L}_{-} 1\right)$.

2.3. Analytical methods

The samples were analyzed immediately or were stored at cold

temperature $\left(4 \_\right.$) prior to the analysis. The samples for the determination

of dissolved components, e.g., ammonium, nitrate, and

nitrite concentration, were analyzed using an Advanced Compact

Ion Chromatograph IC 861 (Metrohm_Ltd., Herisau, Switzerland)

and guard column. The effluents used to determine the cations

and anions was prepared using ultra pure water (18.2 ls) containing

pyridine-2,6-dicarbonsaure (0.117 $\mathrm{g} \mathrm{L}_{-}$) and $\mathrm{HNO}_{3}(0.11$

$\mathrm{mL} \mathrm{L}_{-}$) for cation components, and $\mathrm{NaCO}_{3}\left(0.3392 \mathrm{~g} \mathrm{~L}_{-}{ }_{1}\right), \mathrm{NaHCO}_{3}$

(0.084 $\left.\mathrm{mL} \mathrm{L}_{-} 1\right)$, and $\mathrm{H}_{2} \mathrm{SO}_{4}(0.1 \mathrm{M})$ for anion components, respectively. Samples were filtered before the analysis using a

$0.2 \mathrm{~lm}$ filter. Temperature, $\mathrm{pH}$, and $\mathrm{DO}$ were monitored continuously

online. In addition, other experimental tests such as MLSS,

mixed liquor volatile suspended solids (MLVSS), were determined

using standard methods (APHA et al., 2005). The repetition of analysis

was considered when an error higher than $5 \%$ was observed in

the samples during the experiment. The ability of system for

ammonium removal can be expressed in term of current efficiency

(CE) according to Eq. (3) (Prosnansky et al., 2002; Ghafari et al.,

2009).

Full text is available at :

http://ac.els-cdn.com/S0960852412012655/1-s2.0-S0960852412012655-main.pdf? tid=ff6a1412-9a09-11e3-

aa09-00000aacb360\&acdnat $=139288552829 f 0 c 3 b e 0 c 04871761$ a134e400240902

http://www.sciencedirect.com/science/article/pii/S0960852412012655 